\title{
Cholinergic Agonist Carbachol Enables Associative Long-Term Potentiation in Piriform Cortex Slices
}

\author{
MADHVI M. PATIL, CHRISTIANE LINSTER, EUGENE LUBENOV, AND MICHAEL E. HASSELMO \\ Department of Psychology, Harvard University, Cambridge, Massachusetts 02138
}

\begin{abstract}
Patil, Madhvi M., Christiane Linster, Eugene Lubenov, and Michael E. Hasselmo. Cholinergic agonist carbachol enables associative long-term potentiation in piriform cortex slices. J. Neurophysiol. 80: 2467-2474, 1998. Pyramidal cells in piriform (olfactory) cortex receive afferent input from the olfactory bulb as well as intrinsic association input from piriform cortex and other cortical areas. These two functionally distinct inputs terminate on adjacent apical dendritic segments of the pyramidal cells located in layer Ia and layer Ib of piriform cortex. Studies with bath-applied cholinergic agonists have shown suppression of the fast component of the inhibitory postsynaptic potentials (IPSPs) evoked by stimulation of the association fibers. It was previously demonstrated that an associative form of LTP can be induced by coactivation of the two fiber systems after blockade of the fast, $\gamma$-aminobutyric acid-Amediated IPSP. In this report, we demonstrate that an associative form of long-term potentiation can be induced by coactivation of afferent and intrinsic fibers in the presence of the cholinergic agonist carbachol.
\end{abstract}

INTRODUCTION

Olfactory (piriform) cortex was previously modeled as an associative memory network (Haberly 1985; Haberly and Bower 1989; Hasselmo 1993; Hasselmo and Bower 1993). Pyramidal cells in piriform cortex receive distributed afferent input from the olfactory bulb on their distal dendrites. In addition, pyramidal cells are highly interconnected via an extensive association fiber system. The spatial segregation of the two fiber systems (layer Ia receives afferent input, layer $\mathrm{Ib}$ association fiber input) makes this system particularly attractive for the study of mechanisms underlying memory formation and retrieval. More recently, the importance of cholinergic modulation in associative memory function has been shown theoretically (Barkai and Hasselmo 1994; Hasselmo 1993). These theoretical results were based on cholinergic effects on excitatory synaptic transmission, pyramidal cell adaptation, and enhancement of long-term potentiation (LTP) in the association fiber layer (Barkai and Hasselmo 1994; Hasselmo and Barkai 1995; Hasselmo and Bower 1992). Here we add a new finding to the various mechanisms underlying the role of cholinergic modulation for the associative memory function in olfactory cortex. The existence of associative LTP between the afferent fiber layer and the association fiber layer was suggested previously (Kanter and Haberly 1993); we show that this form of associative LTP can be induced in the presence of the cholinergic agonist carbachol.

Experimental results have shown that $N$-methyl-D-aspartate (NMDA)-dependent synaptic plasticity exists in both the afferent (Jung et al. 1990) and association fiber system
(Kanter and Haberly 1990). These experiments used the standard experimental protocol for induction of LTP in brain slice preparations of the hippocampus (Madison et al. 1991). More recently, Kanter and Haberly (1993) have shown that another form of LTP, associative LTP, can be induced between the afferent and the association fiber layer when the fast, chloride-mediated inhibition is blocked. In the associative LTP paradigm, a weak, (nonpotentiating) stimulus to one set of fibers can produce potentiation if paired with a strong (potentiating) stimulus to the second set of fibers (Barrinuevo and Brown 1983; Levy and Steward 1979). In piriform cortex, this form of associative LTP between afferent input (in layer Ia) and association fibers (in layer Ib) could only be obtained when chloride-mediated inhibition was blocked (Kanter and Haberly 1993).

Experiments in our laboratory have shown a strong, selective suppression of the fast, chloride-mediated inhibition in layer Ib by the cholinergic agonist carbachol (Patil and Hasselmo 1996, 1997; Patil et al. 1997a). In this report, we show that the cholinergic suppression of the fast inhibition by bath application of carbachol is sufficient to allow associative LTP of association fibers during strong stimulation of afferent fibers and weak stimulation of association fibers similar to that described by Kanter and Haberly (1993) under blockade of chloride-mediated inhibition. In contrast to the results described by Kanter and Haberly (1993), in which blockade of chloride-mediated inhibition allowed associative LTP to occur in either direction, carbachol did not enhance the potentiation of afferent fibers during strong stimulation of association fibers and weak stimulation of afferent fibers. This further supports our hypothesis that the laminar suppression of inhibition caused by carbachol facilitates this type of potentiation. A preliminary report of these results was published previously in abstract form (Patil et al. 1997b).

\section{METHODS}

Extracellular field potential recordings were obtained from layers $\mathrm{Ia}$ and $\mathrm{Ib}$ of the piriform cortex in the in vitro slice preparation. Slices $400 \mu \mathrm{m}$ thick were obtained from adult female SpragueDawley rats (150-200 g) with standard procedures (Hasselmo and Bower 1992) in accordance with institutional guidelines. The animal was lightly anesthetized with halothane and decapitated. The brain was rapidly removed and placed in chilled oxygenated artificial cerebrospinal fluid (ACSF) maintained close to $4^{\circ} \mathrm{C}$. Slices were cut in the coronal plane, perpendicular to the laminar organization of the piriform cortex, with a vibratome. Once cut, the slices were stored at room temperature in a chamber containing oxygenated ACSF solution with the following composition (in 
A
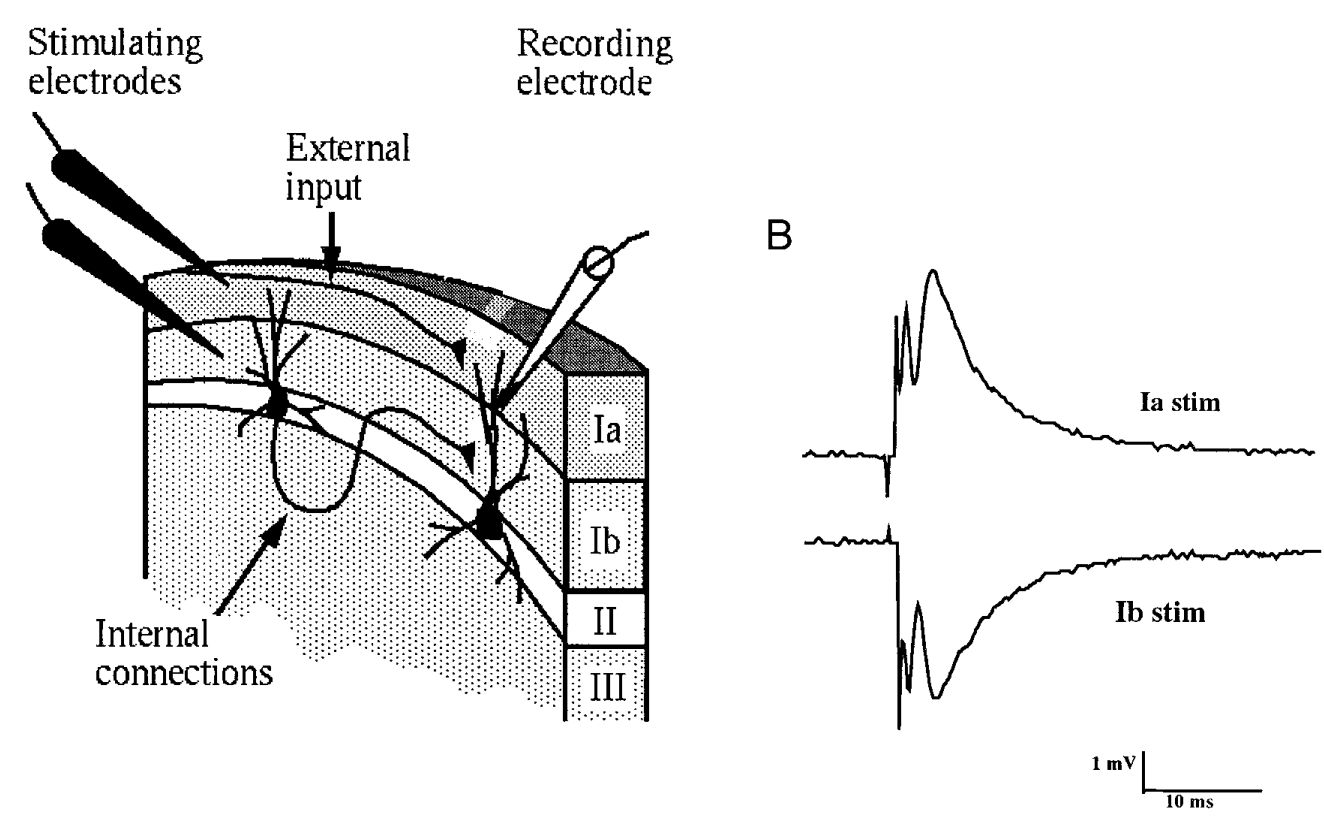

C

D
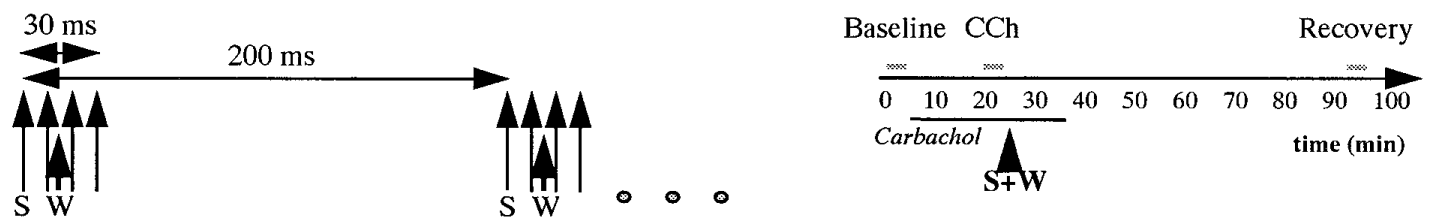

FIG. 1. Schematic representation of the brain slice preparation of piriform cortex and the experimental protocol. A: stimulating electrodes were placed among afferent fibers from the lateral olfactory tract in layer Ia and among association fibers in layer Ib under visual guidance. Extracellular recording electrodes were placed at the boundary of the two layers. $B$ : potentials recorded in response to stimulation of layer Ia (afferent fibers) and layer Ib (association fibers) at the boundary of layers Ia and Ib. C: potentiation trains in layer Ia consisted of 10 sets of 4 pulses $(100 \mathrm{~Hz}, \mathrm{~S})$ at $200-\mathrm{ms}$ intervals. During pairing of strong (S) and weak (W) stimuli, weak test pulses in layer Ib were delivered at 200-ms intervals between the second and third pulse of the four pulse burst. In experiments with multiple pairings, 3 consecutive pairings of weak and strong stimuli were delivered at 5-min intervals. $D$ : experimental protocol. Weak stimuli in layer Ib were delivered continuously throughout the experiment at 30 -s intervals. Baseline response to layer $\mathrm{Ib}$ test pulses were recorded at the beginning of the experiment. Approximately 20 min after the beginning of carbachol application a single or 3 pairings of potentiation trains with weak stimuli were delivered $(S+W)$. After washout, the response to test stimuli in artificial cerebrospinal fluid (ACSF) was recorded for $\geq 60 \mathrm{~min}$. For analysis, 10 consecutive trials were averaged $5 \mathrm{~min}$ after the beginning of the experiment (Baseline), 20 min after the beginning of carbachol perfusion (CCh), and 40-45 min after the beginning of washout (Recovery).

$\mu \mathrm{M}): 124 \mathrm{NaCl}, 5 \mathrm{KCl}, 1.2 \mathrm{KH}_{2} \mathrm{PO}_{4}, 1.3 \mathrm{MgSO}_{4}, 2.4 \mathrm{CaCl}_{2}, 26$ $\mathrm{NaHCO}_{3}$, and $10 \mathrm{D}$-glucose, with a pH of 7.4-7.5.

After $1 \mathrm{~h}$ of incubation at room temperature slices were placed on a nylon mesh in a submerged chamber with oxygenated ACSF flowing over the slices at a rate of $1 \mathrm{ml} / \mathrm{min}$. A thermistor, placed just below the mesh, monitored and controlled the temperature of the ACSF through a temperature regulator circuit, maintaining temperature at $34^{\circ} \mathrm{C}$. Extracellular recordings were taken with glass pipettes of $\sim 5 \mathrm{M} \Omega$ impedance and filled with $3 \mathrm{M} \mathrm{NaCl}$. The orthodromic stimuli were delivered through a NeuroData PG4000 stimulator, with two fine unipolar tungsten electrodes. Stimulation electrodes were placed in layers $\mathrm{Ia}$ and $\mathrm{Ib}$ under direct visual guidance. The recording electrode was placed in the boundary between the two layers. The configuration of the brain slice preparation is schematically shown in Fig. $1 \mathrm{~A}$. Potentiating (strong) pulses were delivered to layer Ia and single (weak) pulses were delivered to layer Ib. Figure $1 B$ shows the response to stimulation in layers $\mathrm{Ia}$ and $\mathrm{Ib}$ as recorded in the boundary between the two layers.

\section{Stimulation protocol (Fig. 1C)}

LAYER Ib TEST STIMULI. After the protocol of Kanter and Haberly (1993), test stimulus in layer Ib was 0.1-ms shocks delivered at intervals of $30 \mathrm{~s}$. Shock strength for test pulses was adjusted to give a response amplitude of $\sim 50 \%$ of the maximal response.

LAYER Ia POTENTIATION TRAINS. Pulses using near maximal strength (strong stimuli) were used for potentiating trains in layer Ia. Potentiating trains consisted of 10 sets of 4 pulses at $100 \mathrm{~Hz}$ delivered at 200-ms intervals.

PAIRING OF STRONG AND WEAK STIMULI. During pairing of strong and weak stimuli, test stimuli in layer $\mathrm{Ib}$ were given at 5$\mathrm{Hz}$ frequency between the second and third pulse of the four pulse burst in layer Ia, as shown in Fig. $1 C$. Two different experimental protocols were used: 1) a single pairing of potentiating trains and weak stimuli and 2) three repetitive pairings of strong and weak stimuli at 5-min intervals. 


\section{Experimental protocol (Fig. 1D)}

The baseline response to test pulses in layer $\mathrm{Ib}$ was recorded at the beginning of the experiment (50\% of the maximal strength). Subsequent responses were then expressed as a percent of this baseline. Test stimuli in layer $\mathrm{Ib}$ were delivered throughout the experiment at 30-s intervals. After baseline amplitude was determined, the slice chamber was perfused with carbachol $(50,10$, or $1 \mu \mathrm{M})$. After stabilization of the response in carbachol (20-25 min after beginning of carbachol perfusion), either a single or three repetitive pairings of strong and weak stimuli were delivered. Washout started $15 \mathrm{~min}$ after the beginning of the first pairing and continued for $\geq 45 \mathrm{~min}$. To test for the healthiness of the slice, at the end of the experiment, the stimulation strength was once more adjusted to the amplitude that gave rise to the maximal response (which was $200 \%$ of baseline) at the beginning of the experiment. Slices that failed to reach $\geq 180 \%$ of the baseline response were excluded from the analysis.

\section{Analysis}

Amplified responses to test stimuli in layer $\mathrm{Ib}$ were recorded and stored by computer. In a later analysis phase, the onset slope of individual recordings was calculated (measured between 2 points in the linear rising phase of the population excitatory postsynaptic potential).

BASELINE. The baseline response (Baseline in Fig. 1D) was calculated as the average response of 10 test stimuli 5 min after the beginning of recording in ACSF. All other responses were then expressed as percent of baseline. Results are reported as the average value \pm SE. Significance of the reported effects was calculated by comparing the recovery values with the baseline values for all slices with a paired $t$-test $(P<0.05)$.

CARBACHOL. The response to layer Ib stimulation after carbachol perfusion ( $\mathrm{CCh}$ in Fig. $1 \mathrm{D}$ ) was recorded as the average response to 10 sequential test stimuli $15 \mathrm{~min}$ after the beginning of carbachol perfusion.

RECOVERY. The response to layer Ib stimulation after washout of carbachol (Recovery in Fig. 1D) was recorded as the average response of 10 sequential trials $40-45 \mathrm{~min}$ after the beginning of washout.

\section{Control experiments}

For control purposes, the following experiments were performed with the experimental protocol described previously; (control 1) $10 \mu \mathrm{M}$ scopolamine was perfused into the bath solution simultaneously with $50 \mu \mathrm{M}$ carbachol, (control 2) the stimulation pattern of the experiment was performed without carbachol perfusion, (control 3) the experiment was performed with test stimuli at 30$\mathrm{s}$ intervals at all times during baseline, carbachol, and washout phases (i.e., no presentation of weak and strong stimuli), (control 4 ) the experiment was performed with only the weak 5-Hz stimulation in the presence of carbachol (i.e., no presentation of strong stimuli), and (control 5) paired weak and strong stimuli in ACSF, unpaired strong stimuli in $\mathrm{CCh}$, and paired weak and strong stimuli in $\mathrm{CCh}$ were performed sequentially in the same slice. These control experiments were performed to insure that neither the pairing of weak and strong stimuli nor carbachol perfusion alone is sufficient to induce potentiation of the layer Ib response.

\section{RESULTS}

As described by Kanter and Haberly (1993) under control conditions, potentiating stimuli in layer Ia did not induce LTP in the association fiber pathway. Similarly, under con- trol conditions (in ACSF), pairing of strong and weak stimuli did not induce LTP in the association fiber pathway.

As described previously (Hasselmo and Bower 1992), bath application of $50 \mu \mathrm{M}$ carbachol selectively suppresses excitatory synaptic potentials evoked by layer Ib stimulation. In our experiments, after bath application of $50 \mu \mathrm{M}$ carbachol, the observed response to weak stimulation in layer $\mathrm{Ib}$ decreased to $70.3 \pm 3.2 \%(n=18)$ of the control response.

\section{Single pairings}

In 10 slices, a single pairing of strong and weak stimuli was applied. In these slices the average response to layer $\mathrm{Ib}$ stimulation after washout increased to $133.78 \pm 1.52 \%(n=$ $10)$ of baseline. This increase was highly significant $(P<$ $0.0001)$. The response increase was measured $\sim 55-65 \mathrm{~min}$ after the application of the potentiating stimulus train. Figure $2 A$ shows the time course of an experiment with a single pairing of strong and weak stimuli.

\section{Multiple pairings}

In eight slices, three pairings of strong and weak stimuli were delivered in 5-min intervals. In these slices the average response to layer $\mathrm{Ib}$ stimulation after washout increased to $156.58 \pm 3.2 \%(n=8)$ of baseline. This increase was highly significant $\left(P<10^{-5}\right)$. Figure $2 B$ shows the time course of an experiment with three consecutive pairings of weak and strong stimuli.

Figure $2 C$ summarizes the average results for all the slices and compares these results with results in scopolamine, and Fig. $2 D$ shows examples of responses to stimulation of layer $\mathrm{Ib}$ recorded at the beginning of the experiment (baseline) and after washout (recovery).

\section{Dose dependency of the multiple-pairing effect}

In five slices, three pairings of strong and weak stimuli were delivered in $10 \mu \mathrm{M} \mathrm{CCh}$. In these slices the average response to layer $\mathrm{Ib}$ stimulation after washout increased to $127.68 \pm 3.17 \%(n=8)$ of baseline. This increase was much lower than the increase observed in $50 \mu \mathrm{M} \mathrm{CCh}$. In an additional three slices, three pairings were delivered in the presence of $1 \mu \mathrm{M} \mathrm{CCh}$. In these slices, an increase to $147.34 \pm 14.2 \%(n=3)$ of baseline was observed after washout, which was much larger than the increase observed at the higher doses of $10 \mu \mathrm{M}$ but lower than the increase observed with $50 \mu \mathrm{M}$. The larger effect at lower concentrations probably involves a separate mechanism, as LTP without associative stimulation has been shown with perfusion of low doses $(\leq 1 \mu \mathrm{M})$ of carbachol in hippocampal slice preparations (Auerbach and Segal 1994). That previous study did not show cholinergic enhancement of LTP at higher concentrations of carbachol. This is consistent with our finding that higher concentrations of carbachol do not cause LTP in the absence of higher frequency repetitive stimulation.

\section{Muscarinic nature of the effect}

To test for the muscarinic nature of the observed effect of $\mathrm{CCh}$, we perfused $10 \mu \mathrm{M}$ scopolamine simultaneously 
A

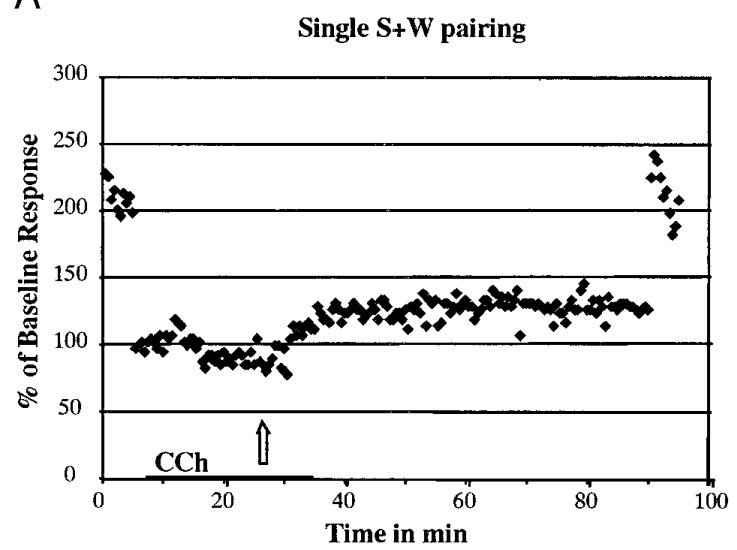

C

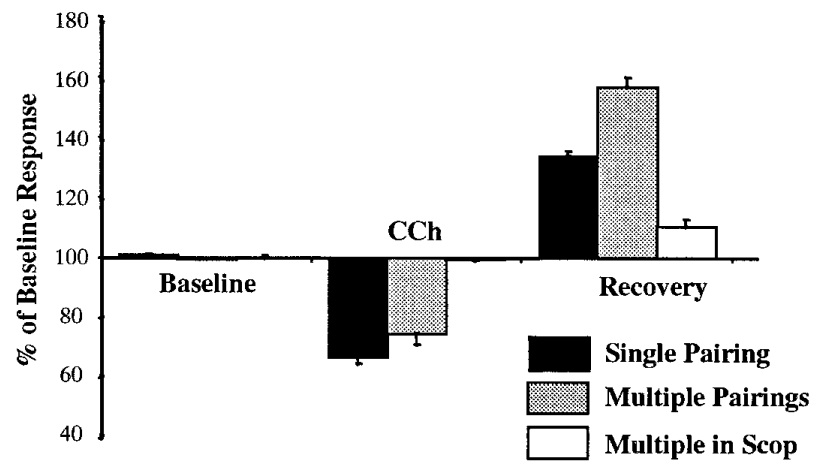

B

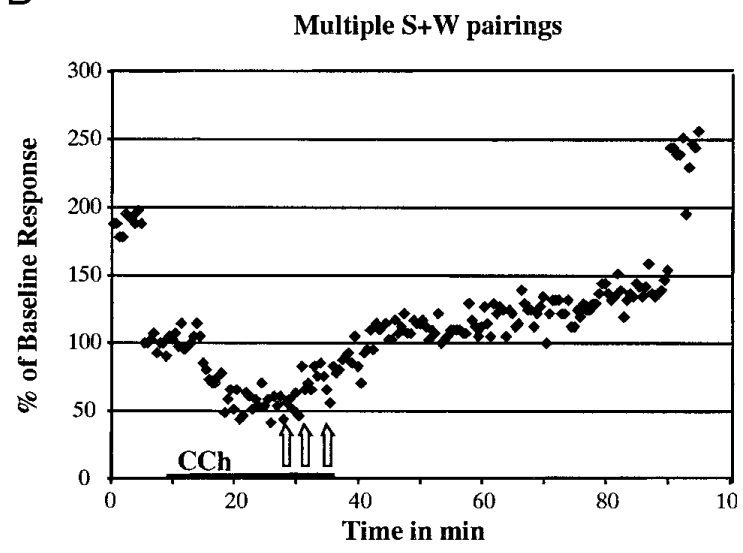

D

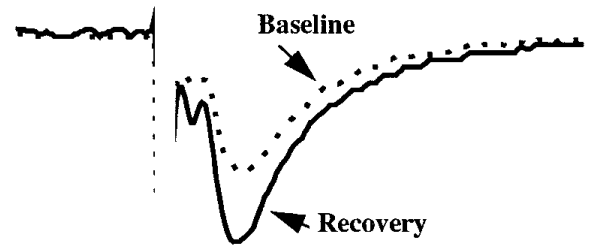

FIG. 2. Summary of results at $50 \mu \mathrm{M}$ carbachol. $A$ and $B$ : time course of an experiment with a single pairing $(A)$ and multiple pairings $(B)$ of strong and weak stimuli. Onset slopes of potentials elicited by layer Ib test pulses are shown as percent of baseline. At the beginning of the experiment, the maximal response to layer Ib stimulation was recorded. Stimulation strength was then adjusted to evoke $\sim 50 \%$ of the maximal response. After bath application of carbachol, the response amplitude to test stimuli decreased and stabilized after $\sim 20-30 \mathrm{~min}$. Pairing of strong and train weak stimuli (arrow) resulted in an increase of the response to test stimuli in layer Ib. After washout, the response to test stimuli in layer Ib is significantly higher compared with the baseline recorded at the beginning of the experiment. At the end of the experiment, stimulation strength is adjusted to the maximal response strength recorded at the beginning of the experiment. $C$ : average responses recorded in experiments using 1$)$ single pairings in $50 \mu \mathrm{M} \mathrm{CCh}(n=10), 2)$ multiple pairings in $50 \mu \mathrm{M} \mathrm{CCh}(n=10)$, and 3) multiple pairings in $50 \mu \mathrm{M} \mathrm{CCh}$ and $10 \mu \mathrm{M}$ scopolamine $(n=7)$. Onset slopes are given as percent of baseline. Error bars indicate SEs. CCh: average response 20-30 min after application of 50 $\mu \mathrm{M}$ carbachol; Recovery: average response recorded 40-45 min after the beginning of washout. $D$ : potentials in response to layer $\mathrm{Ib}$ stimulation. Each trace is an average of 5 recorded potentials. Response recorded in ACSF before application of potentiating stimulus (dashed line) and response recorded $60 \mathrm{~min}$ after beginning of washout (solid line).

with $50 \mu \mathrm{M}$ CCh in seven slices. In these slices, there was no significant increase $(P>0.1)$ of the response after washout (110.32 $\pm 2.3 \%$ of baseline; $n=7$; Fig. $2 C$, white bars $)$.

CONTROL EXPERIMENTS. In a first control experiment, in eight slices, we applied the experimental protocol described previously (with multiple pairings of strong and weak stimuli) but did not perfuse carbachol into the bath. In these slices, the average response 55-60 min after multiple pairings of weak and strong stimuli was $106.23 \pm 3.0 \%(n=$ $8)$ of baseline. There was no significant difference $(P>0.2)$ between the baseline responses and the responses after the potentiating stimuli. Figure $3 A$ shows the time course of a control experiment in ACSF, and Fig. $3 B$ summarizes the average responses measured. The average responses of the ACSF control (Fig. 3B, solid bars) are similar to those obtained with simultaneous perfusion of scopolamine and carbachol described previously (Fig. $3 B$, shaded bars).

In a second control experiment, in four slices we observed the potentials evoked in layer Ib without potentiating stimuli. After carbachol perfusion, a stable baseline of the potential was obtained after 20-30 min. After washout, a stabilization occurred at $\sim 30 \mathrm{~min}$. As described before (Hasselmo and Bower 1992), in recovery the responses returned to $89.23 \pm$ $4.15 \%$ of the baseline response. This shows that perfusion of carbachol by itself at this concentration does not cause the potentiation of the response to layer Ib stimulation. Figure $3 C$ shows the time course of this control experiment, and Fig. $3 D$ summarizes the average responses measured in this control experiment.

In a third control experiment, we performed sequential controls in the same slice $(n=5)$. In keeping with Kanter and Haberly (1993), a tetanus was given in ACSF, followed by a pairing of weak and strong stimuli in ACSF. Five minutes after the pairing, $50 \mu \mathrm{M}$ carbachol was perfused into the bath. Approximately $20 \mathrm{~min}$ after the beginning of carbachol perfusion, a tetanus was applied, followed by a 
A

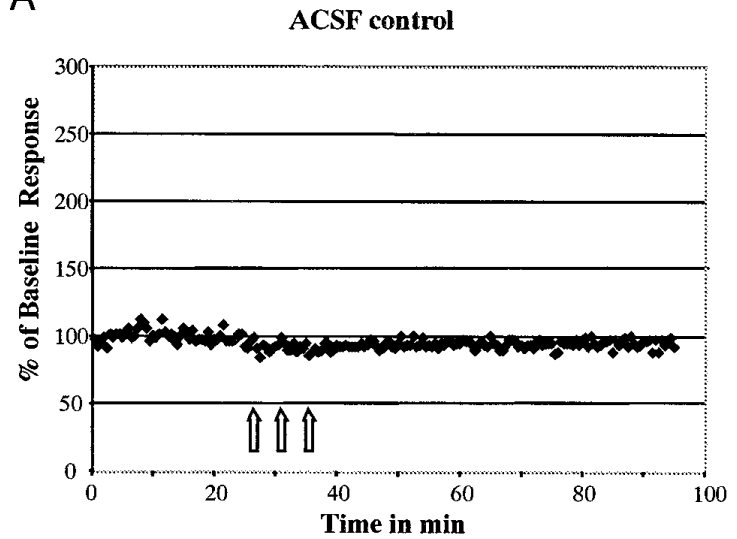

B

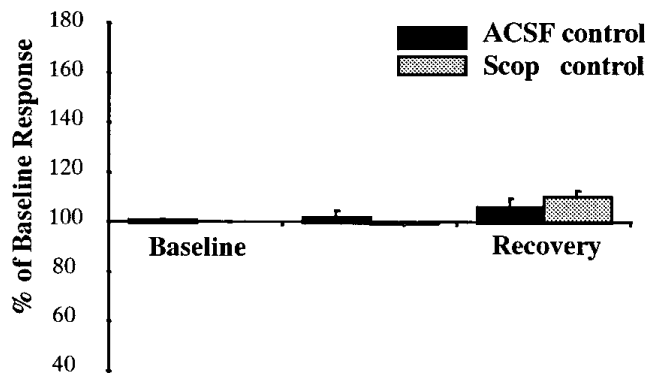

C

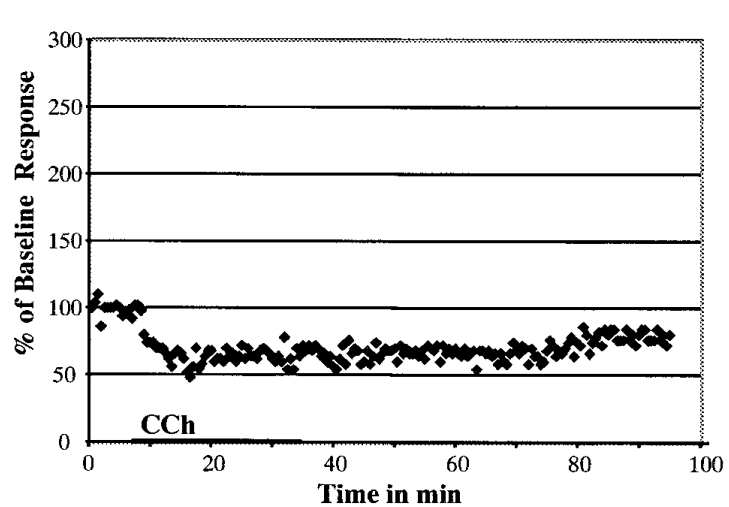

D

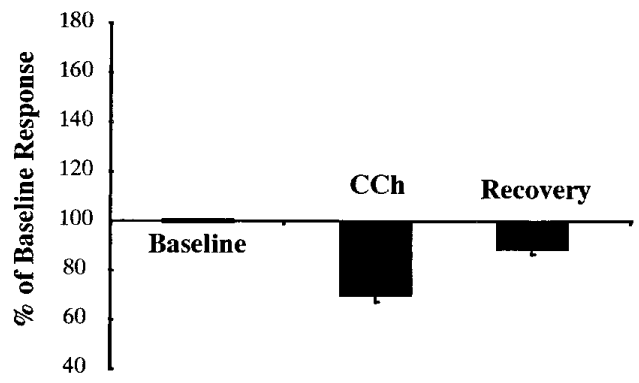

FIG. 3. Summary of control experiments. A: time course of a control experiment in ACSF. Onset slopes of potentials elicited by layer $\mathrm{Ib}$ test pulses are shown as percent of baseline. As in Fig. 2, multiple pairings were delivered 25 min after the beginning of the experiment; $60 \mathrm{~min}$ after the pairing of strong and weak stimuli, no significant change in the response to layer Ib stimulation was observed. $B$ : average responses recorded in 8 slices under ACSF and under CCh + scopolamine (same data as in Fig. $2 C$ shown here for comparison purposes). Onset slopes are given as percent of baseline; error bars indicate SEs. $C$ : time course of a control experiment in $50 \mu \mathrm{M}$ carbachol with continuous stimulation of layer $\mathrm{Ib}$ but no pairing of strong and weak stimuli. Onset slopes are shown as percent of baseline. Multiple tetani were delivered 20-30 min after the beginning of carbachol application; $60 \mathrm{~min}$ after washout, no significant increase in the response to layer $\mathrm{Ib}$ stimulation compared with baseline was observed. $D$ : average responses recorded in 8 slices under ACSF. Onset slopes are given as percent of baseline; error bars indicate SEs.

pairing of weak and strong stimuli after $10 \mathrm{~min}$. Wash began $\sim 10$ min after the pairing (Fig. 4A). We observed no potentiation after the paired stimulation in ACSF $(107 \pm 5.1 \%$ of baseline; LTP-ACSF in Fig. $4 B$ ) or after the tetanus in carbachol. A strong potentiation could always be observed after the paired stimulation in CCh $(188 \pm 2.0 \%$ of baseline; LTP-CCh in Fig. 4B). After wash, the increase of the response was similar to that reported above for single pairing experiments ( $136 \pm 0.9 \%$ of baseline; Recovery in Fig. $4 B$ ).

POTENTIATING STIMULI IN LAYER Ib PAIRED WITH WEAK STIMULATION IN LAYER Ia. In five slices, we reversed the stimulation patterns in the two layers. Layer Ia now received the weak stimulation, and the strong stimulus tetanus was delivered to layer Ib. Figure 5 summarizes the results from this experiment. On the average, when multiple stimulation was used, no significant potentiation of the response to weak stimuli in layer Ia could be observed (113.83 $\pm 4.1 \%$ of baseline; $n=5 ; P>0.2)$. These experiments were performed under $50 \mu \mathrm{M}$ carbachol with the protocol described previously.

\section{IS CUSSION}

These results demonstrate that associative LTP of layer Ib synaptic potentials can be induced in piriform cortex slices when strong stimulation of layer Ia afferent inputs is combined with weak stimulation of layer Ib association fibers in the presence of the cholinergic agonist carbachol. Blockade of the effect of carbachol by scopolamine suggests that the enhancement of associative LTP is due to activation of muscarinic receptors. Previous work has shown that associative LTP can be obtained under similar conditions when $\gamma$ aminobutyric acid $\left(\mathrm{GABA}_{\mathrm{A}}\right)$ inhibition was blocked (Kanter and Haberly 1993). Results from our laboratory (Patil and Hasselmo 1996, 1997; Patil et al. 1997a) recently demonstrated suppression of fast, chloride-mediated inhibition by carbachol in piriform cortex slices. Perfusion of $50 \mu \mathrm{M}$ carbachol in the slice chamber caused a significant suppression $(80 \%)$ of inhibitory synaptic potentials elicited by stimulation of layer $\mathrm{Ib}$ but had a much weaker effect on inhibitory postsynaptic potentials elicited by layer Ia stimulation $(<20 \%$ suppression $)$. Here we have shown that the suppression of inhibition in layer Ib by carbachol is sufficient to obtain associative LTP of association fiber synaptic potentials when strong afferent fiber stimulation was coupled with weak association fiber stimulation (Fig. 2). This resembles results obtained by blockade of fast inhibition with bicuculline (Kanter and Haberly 1993). However, carbachol did not allow associative LTP when the stimulation protocol was 
A

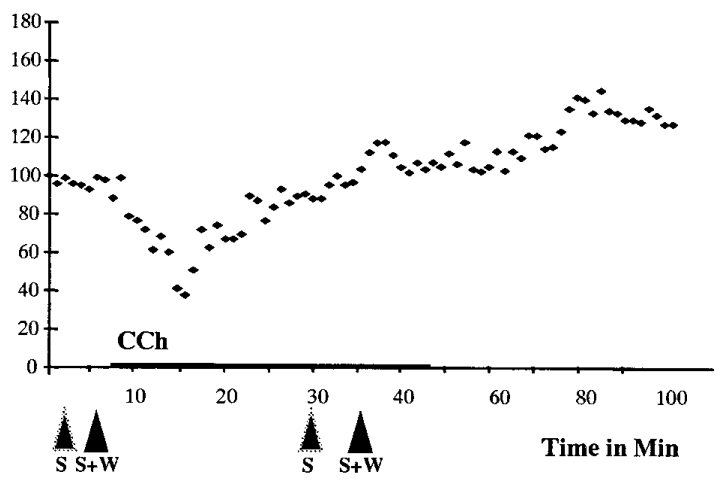

B

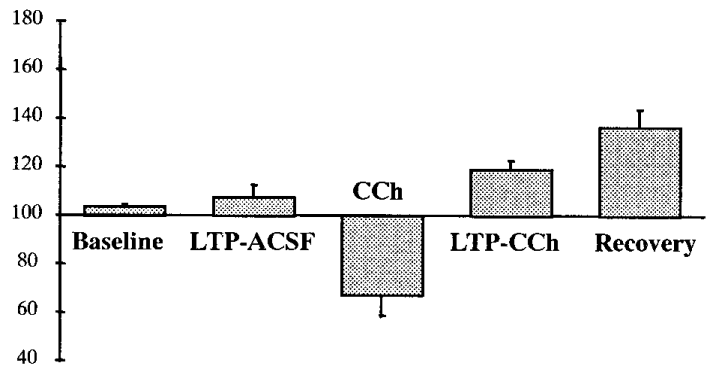

FIG. 4. Experiments with controls and potentiation in the same slice. $A$ : time course of a control experiment in ACSF. Onset slopes are shown as percent of baseline. In ACSF, a single tetanus (S) was given followed by a pairing of strong and weak stimulation $(\mathrm{S}+\mathrm{W})$ after $5 \mathrm{~min} ; 20 \mathrm{~min}$ after the beginning of carbachol perfusion, a second tetanus ( $\mathrm{S}$ ) was given, followed by a single pairing $(\mathrm{S}+\mathrm{W})$ after $\sim 10 \mathrm{~min}$. Washout began 10 min after the pairing. $B$ : average responses recorded in 5 slices. Onset slopes are given as percent of baseline; error bars indicate SEs. Baseline, average response recorded 2 min after beginning of the experiment; LTPACSF, average response recorded after the paired stimulation in ACSF; $\mathrm{CCh}$, average response 15-20 min after the perfusion of carbachol started; $\mathrm{LTP}-\mathrm{CCH}$, average response $5 \mathrm{~min}$ after the paired stimulation in $\mathrm{CCh}$; Recovery, average response $40-45$ min after the beginning of washout.

reversed with respect to the afferent and association fibers (Fig. 5). Kanter and Haberly (1993) reported associative LTP between the two layers independently of which layer received the tetanus when chloride-mediated fast inhibition was blocked. The laminar specificity of the potentiation obtained under carbachol is correlated with the laminar specificity of the cholinergic suppression of inhibition and thus supports our hypotheses. Because piriform cortex receives cholinergic input from the horizontal limb of the diagonal band, this finding demonstrates that associative LTP can be obtained and maintained under physiologically reasonable conditions. In our experiments, only in the presence of carbachol do potentiating stimuli paired with test stimuli lead to an increase in the response to test stimuli. Note that the increase of the response to test stimuli in layer $\mathrm{Ib}$ was observed 40-50 min after washout and 55-65 min after application of the potentiating stimuli.

\section{Modulation of associative LTP}

Kanter and Haberly $(1990,1993)$ proposed that GABA blockade increases the amount of depolarization obtained in the weakly stimulated zone (layer Ib in our experiments), thereby allowing activation of NMDA receptors that are mostly blocked at resting membrane potential. As summarized previously, carbachol decreases inhibitory synaptic transmission in layer Ib by $>80 \%$ (Patil and Hasselmo 1996, 1997), which corresponds to a massive reduction of inhibition in the weakly stimulated zone in our experiment. Similar muscarinic suppression of evoked inhibitory synaptic potentials has been shown in the hippocampal formation (Haas 1982; Pitler and Alger 1992). It has been shown that selective blockade of dendritic $\mathrm{GABA}_{\mathrm{A}}$ inhibition enables expression of the NMDA component in response to burst stimulation in layer Ib (Kanter et al. 1996; Kapur et al. 1997). In piriform cortex, cholinergic agonists enhance LTP obtained by tetanic stimulation in layer Ib (Hasselmo and Barkai 1995); similar effects of cholinergic agonists were reported for hippocampal regions CA1 and dentate gyrus (Blitzer et al. 1990; Burgard and Sarvey 1990; Huerta and Lisman 1994). Carbachol has a number of cellular effects on piriform cortex pyramidal cells, which could presumably facilitate associative as well as nonassociative LTP. These effects include depolarization of pyramidal cells and suppression of pyramidal cell adaptation (Barkai and Hasselmo 1994). In addition to the suppression of inhibitory synaptic potentials, these postsynaptic effects on pyramidal cells may add to the induction of associative LTP, which cannot be obtained in control conditions. Given the fact that Kanter and Haberly (1993) obtained the same amounts of potentiation in their experiments with the $\mathrm{GABA}_{\mathrm{A}}$ antagonist bicuculline, we speculate that the suppression of inhibitory synaptic trans-
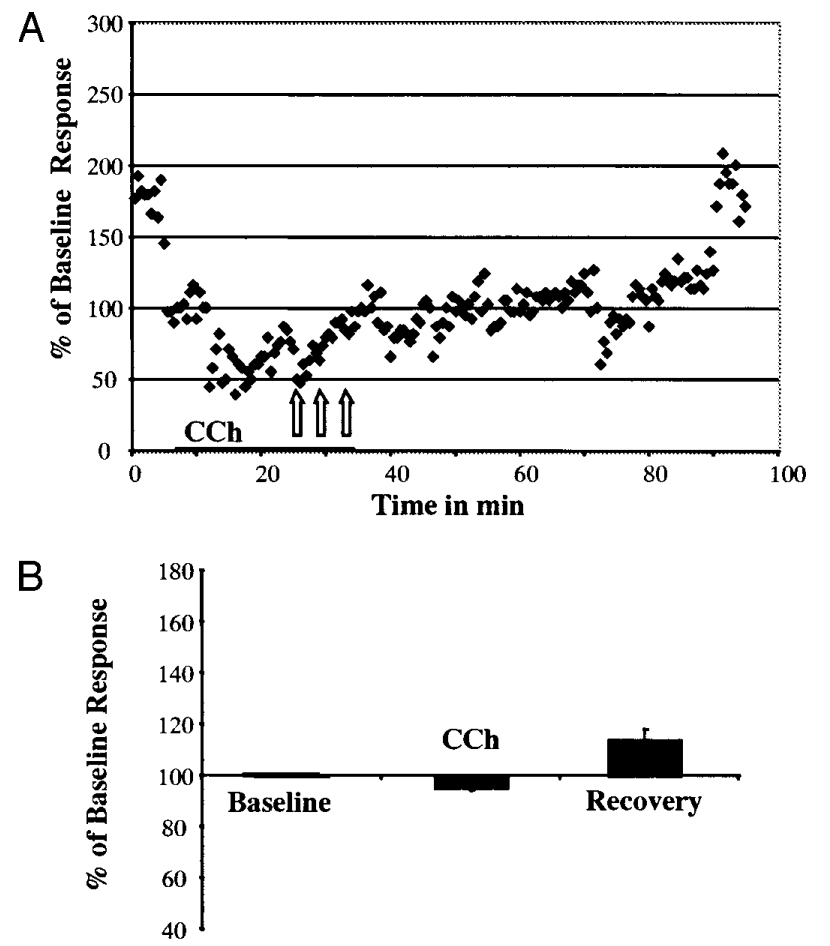

FIG. 5. Summary of results of experiments using weak stimulation in layer Ia paired with tetani in layer $\mathrm{Ib}$. $A$ : time course of an experiment using multiple pairings $(B)$ of strong and weak stimuli in $50 \mu \mathrm{M} \mathrm{CCh}$. This experiment used the stimulation protocol described in METHODS and in Fig. 1, $C$ and $D$, but the stimulation pattern was reversed with respect to layer Ia and layer Ib. Layer Ia received weak stimuli paired with strong stimuli in layer $\mathrm{Ib}$. The onset slopes of the potentials recorded in response to test stimuli in layer Ia are shown as percent of baseline. $B$ : average responses recorded in experiments using multiple pairings in $50 \mu \mathrm{M} \mathrm{CCh}$ $(n=5)$. Onset slopes are given as percent of baseline. Error bars indicate SEs. 
mission by carbachol is the main mechanism underlying the results presented here. This hypotheses is further supported by the laminar specificity of the associative LTP observed here.

\section{Functional significance}

In our models of cortical associative memory function, cholinergic and noradrenergic modulation serves as a regulatory signal that can serve a number of functions, including 1) allowing consistent magnitudes of population activity independent of input to the network (Linster and Hasselmo 1997); 2) switching the network from external input modes appropriate for learning to internal association modes appropriate for retrieval or consolidation (Hasselmo 1993), which can also be described as regulating levels of signal-to-noise ratio (Hasselmo et al. 1997); and 3) enhancing the oscillatory dynamics of piriform cortex circuits (Liljenstrom and Hasselmo 1995). In this context, the results presented in this report add evidence to the role of cholinergic modulation for the switch between learning and recall modes. As stated by Kanter and Haberly (1993), "Control of inhibition is one possible mechanism whereby modulatory neural inputs could determine whether a synaptic network is in a learning receptive state.' Afferent input to layer Ia of piriform cortex represents direct sensory information from the olfactory bulb. In layer Ib, not only association fibers intrinsic to piriform cortex but input from contralateral piriform cortex as well as from other cortical areas converge. The effects of cholinergic agonists in piriform cortex seem to be specific for layer Ib (Hasselmo and Bower 1992; Linster et al. 1997; Patil and Hasselmo 1997), whereas the effects of noradrenaline are less specific to this layer (Hasselmo et al. 1997), suggesting that the segregation of input fibers may be accompanied by a segregation of neuromodulatory inputs. Thus the segregation of afferent and association fiber inputs to different regions may serve to allow associative plasticity to be modulated by specific centrifugal pathways. General suppression of fast inhibition in piriform cortex would lead to epileptiform activity (Kanter and Haberly 1993), whereas a suppression of inhibition only in the dendritic tree would be less destabilizing for the piriform cortex network. We proposed in our modeling work that not only the enhancement of LTP but also the effects on synaptic transmission as well as postsynaptic cellular effects have important consequences for formation and retrieval of memory. For example, enhanced excitability of pyramidal cells may counterbalance the decreased excitatory synaptic transmission observed in our experiments, which may restore the correct initial conditions for the effect on inhibitory synaptic transmission to allow associative LTP. Recent electrophysiological experiments have shown that some pyramidal cells in piriform cortex respond to olfactory stimulation of the ipsilateral and contraleteral naris odor in the same way (Wilson 1997). As it is known that input from the ipsilateral olfactory bulb terminates in layer Ia and input from the contralateral bulb terminates in layer Ib, associative LTP between the two layers may play an important functional role for olfactory learning and memory. Further detailed modeling studies of the piriform cortex will help us to understand the role of the physiological findings presented in this paper in interaction with previous experimental data on cholinergic modulation in the piriform cortex.

Address for reprint requests: C. Linster, Dept. of Psychology, Boston University, 64 Cummington St., Boston, MA 02215.

Received 2 December 1997; accepted in final form 28 July 1998.

\section{REFERENCES}

Auerbach, J. M. And Segal, M. A novel cholinergic induction of longterm potentiation in rat hippocampus. 72: 2034-2040, 1994.

Bark ai, E., Bergman, R. E., Horwitz, G., and Hasselmo, M. E. Modulation of associative memory function in a biophysical simulation of rat piriform cortex. J. Neurophysiol. 72: 659-677, 1994.

BARKAI, E. AND HASSELMO, M. E. Modulation of the input/output function of rat piriform cortex pyramidal cells, J. Neurophysiol. 72: 644-658, 1994.

BarRionuevo, G. AND Brown, T. H. Associative long term potentiation in hippocampal slices. Proc. Natl. Acad. Sci USA 80: 7347-7351, 1983.

Blitzer, R. D., Gil, O., AND Landau, E. M. Cholniergic stimulation enhances lomg term potentiation in the CA1 region of rat hippocampus. Neurosc. Lett. 119: 207-210, 1990.

BuRgard, E. C. AND SARveY, J. M. Muscarinic receptor activation facilitates the induction of long-term potentiation (LTP) in the rat dentate gyrus. Neurosci. Lett. 116: 34-39, 1990.

HaAs, H. L. Cholinergic disinhibition in hippocampal slices of the rat. Brain Res. 233: 200-204, 1982.

HABERLY, L. B. Neuronal circuitry in piriform cortex: anatomy and functional implications. Chem. Senses 10: 219-238, 1985.

HABERLY, L. B and Bower, J. M. Olfactory cortex: model circuit for study of associative memory? Trends Neurosci. 12: 258-264, 1989.

Hasselmo, M. E. Acetylcholine and learning in a cortical associative memory. Neural Comput. 5: 32-44, 1993.

Hasselmo, M. E. AND BARKaI, E. Cholinergic modulation of activity dependent synaptic plasticity in the piriform cortex: brain slice physiology and computational modeling. J. Neurosci. 15: 6592-6604, 1995.

Hasselmo, M. E. AND Bower, J. M. Cholinergic suppression specific to intrinsic not afferent fiber synapses in rat piriform cortex. J. Neurophysiol. 67: 1222-1229, 1992.

Hasselmo, M. E. AND Bower, J. M. Acetylcholine and memory. Trends Neurosci. 16: 218-222, 1993.

Hasselmo, M. E., Linster, C., Patil, M., Ma, D., And Cekic, M. Noradrenergic suppression of synaptic transmission may influence cortical signal-to-noise ratio. J. Neurophysiol. 77: 3326-3339, 1997.

Huerta, P. T. AND Lisman, J. E. Heightened synaptic plasticity of hippocampal CA1 neurons during a cholinergically induced rhythmic state. Nature 364: 723-725, 1994.

JunG, M. W., LARSON, J., AND LYNCH, G. Long-term potentiation of monosynaptic EPSPs in rat piriform cortex in vitro. Synapse 6: 279-283, 1990.

KANTER, E. D. AND HaBerLY, L. B. NMDA-dependent induction of longterm potentiation in afferent and association fiber systems of piriform cortex in vitro. Brain Res. 525: 175-179, 1990.

Kanter, E. D. AND Haberly, L. B. Associative long-term potentiation in piriform cortex slices requires $\mathrm{GABA}_{\mathrm{A}}$ blockade. J. Neurosci. 13: $2477-$ 2482, 1993.

Kanter, E. D., KaPur, A., And Haberly, L. B. A dendritic GABAA mediated IPSP regulates facilitation of NMDA mediated responses to burst stimulation in piriform cortex. J. Neurosci. 16: 307-312, 1996.

Kapur, A., Lytton, W. W., Ketchum, K. L., And Haberly, L. B. Regulation of the NMDA component of EPSP's by different components of postsynaptic GABAergic inhibition: computer simulation analysis in piriform cortex. J. Neurophysiol. 78: 2546-2559, 1997.

LEVY, W. B. AND STEWARD, O. Synapses as associative memory elements in the hippocampal formation. Brain Res. 175: 233-245, 1979.

LilJenstrom, H. AND Hasselmo, M. E. Cholinergic modulation of cortical oscillatory dynamics. J. Neurophysiol. 74: 288-297, 1995.

Linster, C. AND Hasselmo, M. E. Modulation of inhibition in a model of olfactory bulb reduces overlap in the neural representation of olfactory stimuli. Behav. Brain Res. 84: 117-127, 1997.

Linster, C., Wyble, B. P., and Hasselmo, M. E. Reciprocal interactions between the olfactory system and the horizontal limb of the diagonal band of Broca. Soc. Neurosci. Abstr. 23: 807.8, 1997.

Madison, D. V., MalenK A, R. C., AND Nicoll, R. A. Mechanisms underly- 
ing long-term potentiation of synaptic transmission. Annu. Rev. Neurosci. 14: 379-397, 1991 .

Patil, M., Linster, C., And Hasselmo, M. E. Cholinergic modulation of and the role of interneurons in the piriform cortex. In: Computation and Neural Systems, edited by J. M. Bower. New York: Plenum, 1997a.

Patil, M. M. and Hasselmo, M. E. Cholinergic modulation of synaptic inhibition in the piriform cortex. Soc. Neurosci. Abstr. 21: 1824, 1996.
Patil, M. M., Linster, C., Lubenov, E., And Hasselmo, M. E. Cholinergic modulation facilitates LTP in the piriform cortex. Soc. Neurosci. Abstr. 308: 2, 1997b.

Pitler, T. A., AND Alger, B. E. Cholinergic excitation of GABAergic interneurons in the rat hippocampal slice. J. Physiol. (Lond.) 450: 127-142, 1992.

WiLson, D. A. Bi-naral interactions in the rat piriform cortex, J. Neurophysiol. 78: 160-169, 1997. 